\title{
Contre la cigarette électronique
}

\section{Jürg Barben a, Jürg Hammerb}

a Prof. Dr méd., Médecin responsable Pneumologie et Allergologie, Hôpital des enfants de la Suisse orientale, St. Gall

${ }^{b}$ Prof. Dr méd., Directeur Pneumologie et Médecine intensive, Clinique pédiatrique universitaire, Bâle.

\section{Introduction}

Avec l'apparition de nouveaux produits sur le marché, l'épidémie tabagique est clairement devenue plus complexe [1]. Les dix dernières années le marché s'est enrichi de cigarettes électroniques qui miment l'action de fumer par des moyens techniques sans brûler le tabac [2]. Dans ce domaine on doit distinguer entre les appareils qui vaporisent un liquide à l'aide d'un corps de chauffe et ceux qui chauffent le tabac ("heat not burn devices»). Les E-cigarettes furent développées au tournant du siècle pour les fumeurs, en tant qu'alternative au tabac à fumer, ou en tant qu'aide pour l'arrêt du tabagisme. Cependant, leur efficacité pour atteindre ce but n'est que mineur et guère durable [35]. Actuellement les cigarettes électroniques sont promues par des messages publicitaires tels que "pas de combustion", "pas de cendres», "pas d'odeurs de cigarette», et en tant que "meilleure alternative à la cigarette».

\section{Porte d'entrée à l'addiction nicotinique}

Entretemps, la cigarette électronique s'est rapidement répandue parmi la jeunesse, et ce, jusqu'aux cours des écoles. Les E-cigarettes, également appelées par les adolescents "E-shishas», ne ressemblent plus à des cigarettes tabac et sont perçues par les enfants et les jeunes comme des "vaporisateurs» inoffensifs (fig. 1). Par des arômes attractifs et par un marketing intense, les E-shishas sont devenues des produits à la mode, notamment chez la jeunesse suisse. Selon Addiction Suisse, un tiers des 15-24 ans ont déjà essayé une fois une E-cigarette [6]. Beaucoup d'adolescents n'utilisent plus aujourd'hui qu'un seul produit, mais plusieurs préparations (dual use), dont le Snus (tabac oral) et des E-cigarettes [7]. Il y a peu, est apparu sur le marché nord-américain une nouvelle cigarette électronique qui s'est répandu parmi les jeunes grâce à son aspect de stick USB à la mode. Et la firme américaine vise actuellement le marché européen avec ce produit, appelé «Juul» [8]. Aux Etat-Unis les jeunes «vapotent» plus qu'ils ne fument de cigarettes traditionnelles [9].

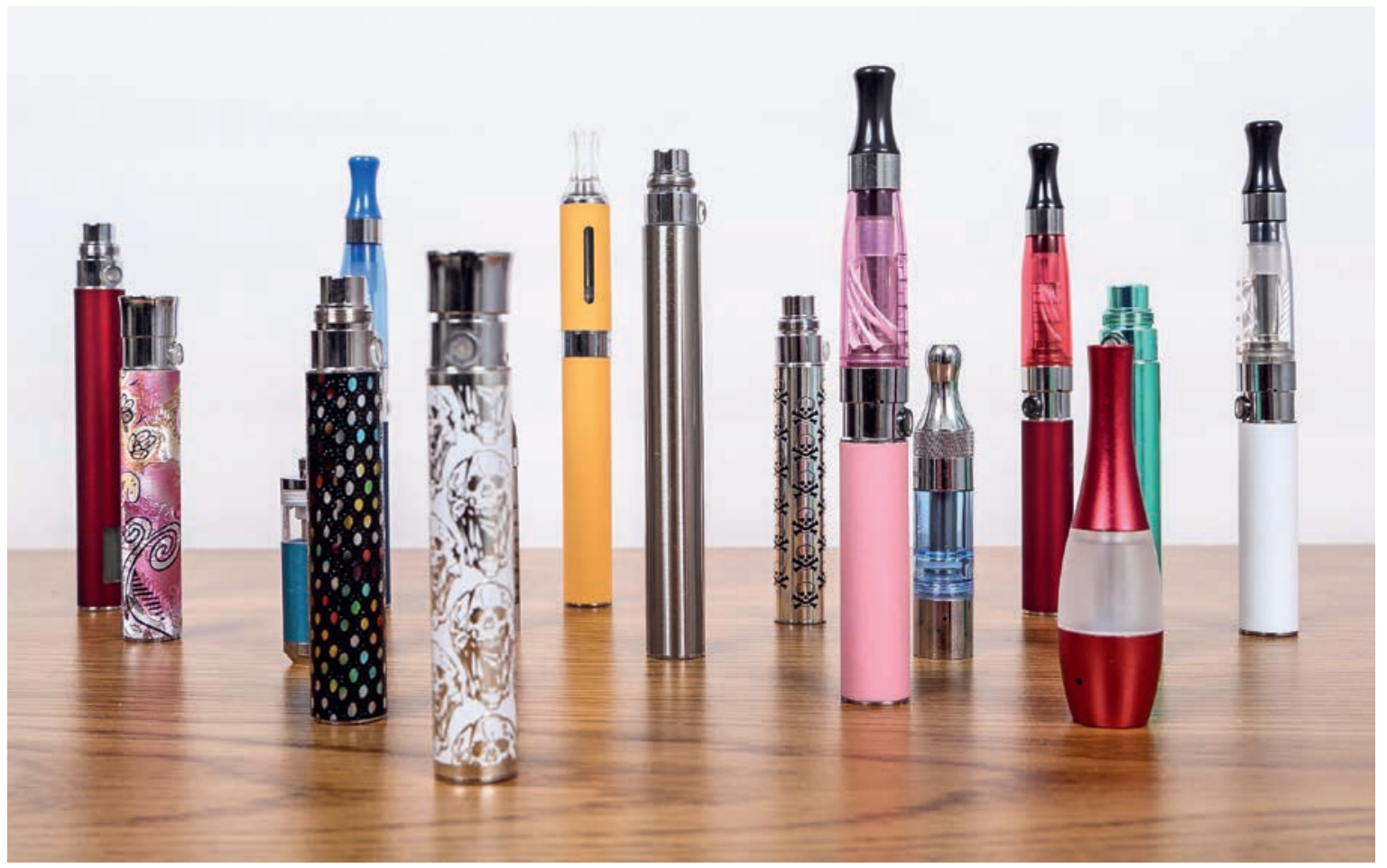

Figure 1: Cigarettes électroniques. 


\section{Vente légale aux mineurs}

Il y a peu, seulement la vente de cigarettes électronique sans nicotine était permise en Suisse. Suite à la plainte d'un producteur, le tribunal administratif fédéral a cassé l'interdiction de l'office fédéral de la sécurité alimentaire et vétérinaire (OFSAV) et depuis mai dernier, les liquides contenant de la nicotine peuvent donc être légalement vendus dans le pays. A cause d'une lacune de la loi, ils sont accessibles également aux mineurs car les E-cigarettes ne tombent actuellement pas dans la catégorie des produits du tabac, mais sous celle des aliments [6]. Sans le savoir, les enfants sont donc exposés très tôt au risque de dépendance à la nicotine. Dépendance de laquelle beaucoup ne pourront pas se libérer et qui assure donc à l'industrie du tabac ses profits [10]. Une méta analyse récente démontre que les jeunes "vapoteurs» présentent un risque 3 à 4 fois plus élevé de devenir fumeur [11].

\section{Nocivité des E-cigarettes}

Selon les données actuelles, l'aérosol des cigarettes électroniques contient moins de substances toxiques et carcinogènes que la fumée de la cigarette conventionnelle, mais les E-cigarettes et E-shishas ne doivent pas pour autant être considérées comme inoffensives [12]. Il manque actuellement des données systématiques de la toxicologie de toutes les substances qui sont inhalées à l'aide d'un brouillard de propylène glycol et de glycérine [2]. Une récente étude de l'université de Berne montre que l'aérosol du tabac chauffé contient les mêmes substances toxiques et carcinogènes que la fumée du tabac conventionnel, et que, par conséquent, on ne peut nullement affirmer que cette "vapeur» est sans danger [13]. Puisqu'il existe aujourd'hui des centaines de liquides pour cigarettes électroniques et parce que leur fabrication n'est standardisée en aucune manière, les études sur leurs aérosols donnent des résultats très variables [14]. Ainsi, la concentration en formaldehyde, un puissant carcinogène, dans l'aérosol, dépend également de la source d'énergie: lorsque le propylène glycol et la glycérine sont chauffés par une tension électrique plus élevée, le formaldehyde est produit à une concentration considérablement plus forte que lors qu'on fume une cigarette conventionnelle [15]. De nombreux métaux lourds ont également été détectés, provenant probablement du corps de chauffe [16]. Avec des expériences en laboratoire, on a prouvé que la "vapeur" de cigarettes électroniques induit des expressions de gènes dans les cellules pulmonaires humaines similaires à celles induites par la fumée des cigarettes conventionnelles
[17]. Les résultats d'études actuelles par des chercheurs indépendants documentent les conséquences diverses sur le poumon, tels que l'hyperréactivité bronchique, la diminution de la défense immune, des nécroses plus nombreuses et la cytotoxicité accrue [18]. Puisque les E-cigarettes ne sont disponibles sur le marché que depuis quelques années, il n'y a pas d'études sur les séquelles à long terme. Mais l'information actuellement à disposition est suffisante pour mettre en garde contre la cigarette électronique en tant que produit qui nuit à la santé.

\section{Des sociétés de spécialistes du poumon mettent en garde contre l'E-cigarette}

Dans cette situation, les médecins spécialistes du poumon lancent actuellement un avertissement. Le Forum of International Respiratory Societies (FIRS) a publié un papier se positionnant sur le sujet des E-cigarettes et des jeunes et la European Respiratory society (ERS) en a publié un sur les produits de tabac à chauffer («heat not burn devices») [19, 20]. Dans la première prise de position, on avertit clairement que les enfants et les jeunes sont particulièrement sensibles de devenir dépendant à la nicotine, ce qui empiète de manière significative sur le développement du cerveau, même si la cigarette électronique ne serait utilisée que de temps en temps [19]. De plus, on prévient que la cigarette électronique doit être considérée comme initiation à fumer du tabac et que ces enfants et jeunes gens ont un risque plus élevé de devenir dépendants au tabac à vie. Les E-cigarettes sont conçues pour être attrayantes pour les enfants et les jeunes et leur publicité et promotion visent à en faire une nouvelle génération de dépendants à la nicotine. Les associations de spécialistes du poumon exigent donc que les E-cigarettes soient strictement régulées comme tous les autres produits du tabac. Cette réglementation devrait comporter une interdiction de la vente aux mineurs, une interdiction des arômes, l'application des mêmes règles de protection contre l'exposition des tiers, similaire à celles contre la fumée passive, ainsi qu'une interdiction globale de la publicité.

Dans la prise de position concernant le tabac chauffé ("heat not burn devices», tel que IQOS et GLO) l'European respiratory society avertit sans ambiguïté qu'on ne peut faire confiance à aucune étude menée ou payée par l'industrie du tabac [20]. Des études indépendantes montrent que de nombreuses substances toxiques et cancérigènes ont été trouvées dans l'aérosol de ces produits, en partie à des concentrations presque identiques à celles de cigarettes conventionnelles. Les affirmations de l'industrie qui avancent que leurs produits 
contiendraient $90-95 \%$ de produits nocifs en moins ne résistent donc pas à un examen indépendant. La société européenne de Pneumologie ne peut pas recommander des produits qui sont nocifs pour le poumon ou pour la santé humaine, et avertit, en conclusion, que les produits chauffés sont également nocifs et rendent dépendant. Ils sabordent par ailleurs le désir des fumeurs d'arrêter leur consommation et présentent une grande tentation pour les non-fumeurs et les mineurs. De plus, les cigarettes électroniques représentent un risque de miner les efforts actuels de prévention et s'opposent à la convention cadre contre le tabagisme de l'OMS (CCLAT), en particulier à la dé-normalisation de la consommation de tabac et à la protection contre la fumée passive. Dans sa conclusion, l'European respiratory society affirme qu'aussi bien les produits de tabac chauffé que les conventionnels, ainsi que le tabac oral tel le Snus, rendent l'être humain dépendant à la nicotine et sont nocifs pour la santé: ils devraient donc être strictement régulé.

\section{Conclusion}

Le fonds de commerce de l'industrie du tabac est la vente de nicotine, une drogue addictive. Pour cette industrie, peu importe que des jeunes gens deviennent dépendants à la nicotine par la cigarette électronique ou conventionnelle, ce qui importe c'est les affaires. Comment pourrait-on expliquer autrement les activités en progression de cette industrie dans le marché de l'E-cigarette? Ce n'est certainement pas que les multinationales du tabac veuillent favoriser l'arrêt du tabagisme chez leurs clients. Les cigarettes électroniques rendent dépendants de la nicotine tout comme les cigarettes traditionnelles, c'est un danger dont il faut protéger nos enfants de manière cohérente. Pour éviter que les mesures de prévention prises jusqu' à présent soient minées par les E-cigarettes et que fumer ne devienne à nouveau un comportement normal, l'engagement de tous les médecins est nécessaire. Il est à espérer que nos politiciens suisses prennent en compte la prise de position des associations de spécialistes des poumons internationaux et européens lors qu'ils discutent et décident de la loi sur les produits du tabac. A défaut, ils devraient s'attendre à ce qu'on leur reproche de se faire les pantins de l'industrie du tabac, qui a toujours et partout défendu ses intérêts par son influence sur les décideurs, pour lutter contre toutes les mesures de santé publique [21-24].

\section{Remerciements}

Les auteurs remercient $M$. Kaelin pour la traduction de l'article original allemand.
Crédit photo

(c) Cherie Moncada | Dreamstime.com

\section{Références}

1 Navas-Acien A. Global Tobacco Use: Old and New Products. Ann Am Thorac Soc 2018; 15(Supplement 2):S69-75.

2 Kaelin R, Barben J, Schuurmans MM. Elektronische Zigaretten, E-Shishas und «heat, but not burn devices». Schweiz Med Forum 2017;17(5):113-9.

3 McRobbie H, Bullen C, Hartmann-Boyce J, Hajek P. Electronic cigarettes for smoking cessation and reduction. Cochrane Database Syst Rev 2014;12:CD010216.

4 Halpern SD, Harhay MO, Saulsgiver K, Brophy C, Troxel AB, Volpp KG. A Pragmatic Trial of E-Cigarettes, Incentives, and Drugs for Smoking Cessation. N Engl J Med 2018;378(24):2302-10.

5 Kalkhoran S, Glantz SA. E-cigarettes and smoking cessation in real-world and clinical settings: a systematic review and meta-analysis. Lancet Respir Med 2016;4(2):116-28.

6 Scheven F. Die E-Zigarette liegt bei Jugendlichen im Trend. Neue Zürcher Zeitung, 15. Juni 2018; S. 24.

7 Soneji S, Sargent J, Tanski S. Multiple tobacco product use among US adolescents and young adults. Tob Control 2016;25(2):174-80 .

8 Schröder T. E-Zigarette Juul will Europa erobern. NZZ am Sonntag, 8. Juli 2018; S. 27.

9 Wang TW, Gentzke A, Sharapova S, Cullen KA, Ambrose BK, Jamal A. Tobacco Product Use Among Middle and High School Students - United States, 2011-2017. MMWR Morb Mortal Wkly Rep 2018:67(22):629-33.

10 Barben J, Runge C. E-Zigaretten und E-Shishas - eine neue Gefahr für unsere Kinder. Pädiatrische Praxis 2016;85(4):525-34.

11 Soneji S, Barrington-Trimis JL, Wills TA, Leventhal AM, Unger JB, Gibson LA et al. Association Between Initial Use of e-Cigarettes and Subsequent Cigarette Smoking Among Adolescents and Young Adults: A Systematic Review and Meta-analysis. JAMA Pediatr 2017;171(8):788-97.

12 Dinakar C, O'Connor GT. The Health Effects of Electronic Cigarettes. N Engl J Med 2016;375(14):1372-81.

13 Auer R, Concha-Lozano N, Jacot-Sadowski I, Cornuz J, Berthet A. Heat-Not-Burn Tobacco Cigarettes: Smoke by Any Other Name. JAMA Intern Med 2018;177(7):1050-2.

14 Stephens WE. Comparing the cancer potencies of emissions from vapourised nicotine products including e-cigarettes with those of tobacco smoke. Tob Control 2018;27:10-7.

15 Jensen RP, Luo W, Pankow JF, Strongin RM, Peyton DH. Hidden formaldehyde in e-cigarette aerosols. $\mathrm{N}$ Engl J Med 2015;372(4):392-4.

16 Olmedo P, Goessler W, Tanda S, Grau-Perez M1, Jarmul S, Aherrera A et al. Metal Concentrations in e-Cigarette Liquid and Aerosol Samples: The Contribution of Metallic Coils. Environ Health Perspect 2018; 126(2):027010-doi: 10.1289/EHP2175.

17) Lee HW, Park SH, Weng MW, Wang HT, Huang WC, Lepor H, et al. E-cigarette smoke damages DNA and reduces repair activity in mouse lung, heart, and bladder as well as in human lung and bladder cells. Proc Natl Acad Sci U S A 2018;115(7):E1560-E1569.

18 Chun LF, Moazed F, Calfee CS, Matthay MA, Gotts JE. Pulmonary toxicity of e-cigarettes. Am J Physiol Lung Cell Mol Physiol 2017;313(2):L193-L2O6.

19 Ferkol TW, Farber HJ, La Grutta S, Leone FT, Marshall HM, Neptune E, et al. Electronic cigarette use in youths: a position statement of the Forum of International Respiratory Societies. Eur Respir J 2018;51(5):Epup ahead. doi: 10.1183/13993003.00278-2018.

20 ERS Tobacco Control Committee. ERS position paper on heated tobacco products. https://www ersnet org/the-society/news/ ers-position-paper-on-heated-tobacco-products. 2018, June 7.

21 Barben J. Tabaklobby und Kinderfänger - wie cool ist rauchen wirklich. Teil 1: Tabakepidemie, Werbung und Manipulation. Schweiz Med Forum 2011;11:370-5.

22 Barben J. Tabaklobby und Kinderfänger - wie cool ist rauchen wirklich. Teil 2: Passivrauchen und Strategien der Tabakindustrie. Schweiz Med Forum 2011;11:389-93.

23 Deutsches Krebsforschungszentrum. Die Tabakindustriedokumente I. Rote Reihe Tabakprävention und Tabakkontrolle [Band 3], 1-78. 2005. Heidelberg.

24 Grüning T, Schönfeld N. Tabakindustrie und Ärzte: «Vom Teufel bezahlt ...». Deutsches Aerzteblatt 2007;104(12):A 770-A 774. 